\title{
Coherent Synchrotron Radiation Analysis for the Photoinjected Energy Recovery Linac and UVFEL Projects at the NSLS
}

\author{
J.H. Wu*, J.B. Murphy, V. Yakimenko, \\ I. Ben-Zvi, W. Graves, E. Johnson, S. Krinsky, T. Shaftan, L.H. Yu \\ NSLS, BNL, Upton, NY 11973, USA
}

\begin{abstract}
Coherent Synchrotron Radiation (CSR) effects in bunch compressors are analyzed. Schemes for reducing the CSR effects are presented.
\end{abstract}

\section{INTRODUCTION}

When an electron bunch is compressed either in a chicane or a bending arc, the CSR will induce an energy redistribution along the bunch. Such energy redistribution will affect the longitudinal emittance as a direct consequence. It will also excite betatron oscillation due to the chromatic transfer functions, and hence a transverse emittance change. For the proposed Photoinjected Energy Recovery Linac (PERL) ${ }^{[1]}$ at the NSLS, bending arcs will be used for bunch compression, while for the UV Free Electron Laser (UVFEL), chicane compressors are used. Since it is normally thought to be deleterious, the CSR effects are analyzed carefully for both projects. It turns out that the CSR effect could be serious, hence schemes to reduce the effect are investigated. Such schemes are incorporated into the PERL arc design. For the UVFEL, chicane parameters are carefully chosen to minimize the CSR effects.

\section{EMITTANCE GROWTH}

In contrast to most collective wake fields, where the leading particles act on the trailing particles, the CSR effects discussed in this paper refer to the field produced by the particles in the tail, which catches the particles in the head. This happens, when the electron trajectory is curved in the bending magnet, since the photon trajectory won't be curved. Analysis of this effect was re-initiated ${ }^{[2,3]}$ in 1995. The longitudinal emittance is affected as a direct consequence. Since the electron bunch is in the dispersive region, the chromatic transfer function will couple this longitudinal energy redistribution into the transverse betatron motion, hence the transverse emittance could grow also. Three reasons of the transverse emittance growth were pointed out ${ }^{[4]}$, 1. The longitudinal energy redistribution is coupled to the transverse phase space by the chromatic transfer function; 2. Centripetal force; 3. Focusing effect. In most of the cases, the first reason dominates, hence it is justified to use the Elegant simulation code ${ }^{[5]}$.

The main points in the CSR analysis could be elucidated by analyzing the 'steady-state' effect. For a bunch with a

\footnotetext{
${ }^{*}$ Also at the C.N. Yang Institute for Theoretical Physics, SUNY Stony Brook, NY 11790-3840
}

Gaussian linear charge density distribution, the energy loss gradient along the bunch is equal to ${ }^{[2,3]}$,

$$
\frac{d \mathcal{E}}{c d t}=\frac{2 N e^{2}}{\sqrt{2 \pi}\left(3 \rho^{2} \sigma_{s}^{4}\right)^{1 / 3}} F\left(\frac{s}{\sigma_{s}}\right),
$$

with

$$
F(\xi)=-\int_{-\infty}^{\xi} \frac{d \xi^{\prime}}{\left(\xi-\xi^{\prime}\right)^{1 / 3}} \frac{d}{d \xi^{\prime}} e^{-\xi^{\prime 2} / 2},
$$

where $N$ is the bunch population, $\rho$ is the bending radius, $\sigma_{s}$ is the rms bunch length. This tells us, when the bunch gets shorter and denser, the CSR effect will be stronger. Plotting $F(\xi)$ shows that the head particles gain some energy while the tail and center part mostly lose. In the $E-t$ phase space, the CSR causes a vertical shearing or chirp as we will further illustrate in Fig. 1.

\subsection{Longitudinal Emittance}

In an arc compressor, particles having higher energy will take a longer path. Hence, we must chirp the bunch such that the head particles have higher energy to compress the bunch; and chirp the other way to decompress it. In the PERL project, the bunch is compressed along the arcs and the bunch is upright in the long straight section, where the insertion devices are located. After that, the bunch is decompressed and so the head particles and tail particles switch role. This leads to the cancellation of the CSR induced energy spread. The mechanism is illustrated in Fig. 1. The momentum compaction in the arc, i.e., $R_{56}$ leads to the horizontal shearing, hence changes the bunch length. Since the CSR effect always causes the head particles to gain energy, while the tail particles lose. Effectively, this is a vertical shearing. During the compression, the head particles are having higher energy, the CSR effect will cause the head particle to further gain energy, hence stretch the bunch in the vertical direction. This leads to the increasing of the energy spread when the bunch is upright. During the decompression, the head particles are having lower energy, the CSR will cause the head particle to gain energy, hence, compress the bunch vertically. This leads to the decrease of the energy spread. Shown in Fig. 2, we find, if there is no CSR effect during the compression, then the ellipse at the middle straight section will be the core part (green). Due to the CSR, it grows (red). During the decompression, the CSR causes the ellipse to shrink to the core part (green). In Fig. 3, $\sigma_{5}$ is the rms bunch length, and $\sigma_{6}$ is the rms local energy spread. We find that when the 
bunch is being compressed, the head particles have higher energy, the energy spread keeps growing due to the CSR vertical stretching, and reaches the peak value at the middle straight section. Then during the decompression, the head particles now have lower energy, the energy spread is effectively reduced due to the CSR effect. In our PERL case, when the bunch entered the compression part of the arc, the local energy spread was $4.66 \times 10^{-4}$. When it comes to the middle straight section, the local energy spread grows to be $5.32 \times 10^{-4}$. After the decompression, the local energy spread reduces to be $4.76 \times 10^{-4}$. This CSR induced energy spread cancellation mechanism ensures the energy recovery.

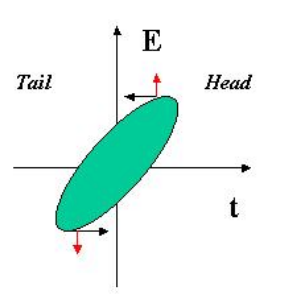

Arc

Compressor

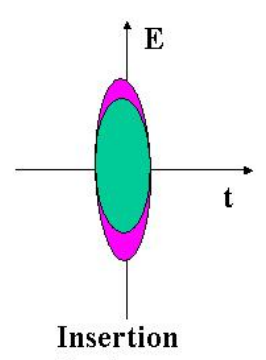

Device

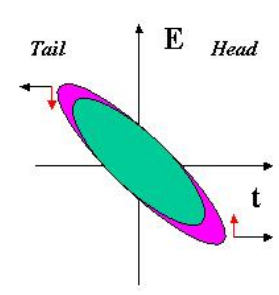

Arc

Decompressor
Figure 1: Longitudinal emittance cancellation mechanism.

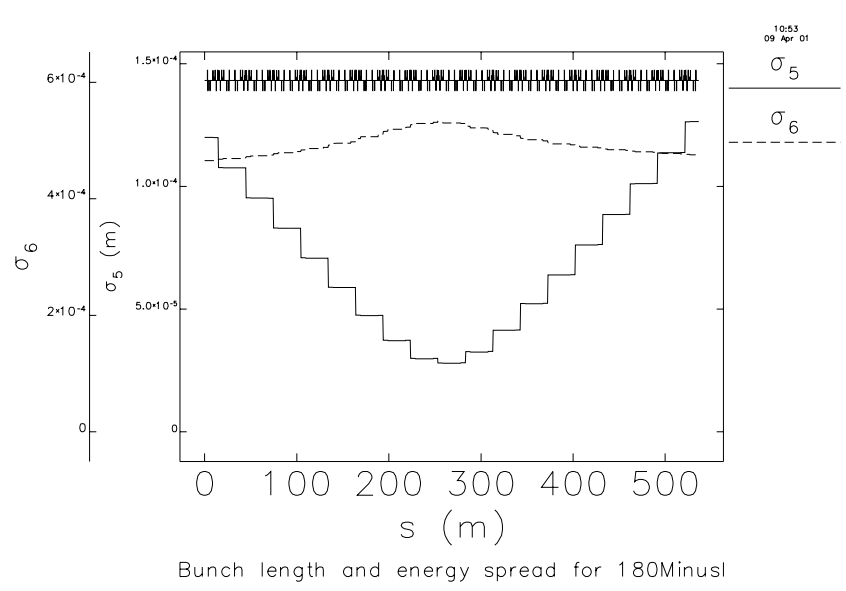

Figure 2: The variation of the bunch length and the energy spread.

The $R_{56}$ in a chicane compressor, has an opposite sign to that in the arc compressor. During the compression, we need to chirp the bunch so that the head particles have lower energy, and the tail particles have higher energy. Due to the CSR effect, the head particles will gain energy, hence the bunch gets compressed also in the vertical direction, therefore, a smaller energy spread.

\subsection{Transverse Emittance}

In the dispersion region, the chromatic transfer function introduces correlations between the transverse phase space and the CSR induced energy spread. The full emittance dilution for the coherent process could be written as ${ }^{[6]}$

$$
\epsilon^{2} \approx \epsilon_{0}^{2}+\epsilon_{0} \frac{1}{\beta}\left[\left\langle\Delta x^{2}\right\rangle+\left(\alpha\left\langle\Delta x^{2}\right\rangle^{\frac{1}{2}}+\beta\left\langle\Delta x^{2}\right\rangle^{\frac{1}{2}}\right)^{2}\right],
$$

with

$$
\left\langle\Delta x^{2}\right\rangle=\left(\int R_{16}(s) \frac{d \sigma_{\delta}}{d s} d s\right)^{2}
$$

and

$$
\left\langle\Delta x^{\prime 2}\right\rangle=\left(\int R_{26}(s) \frac{d \sigma_{\delta}}{d s} d s\right)^{2} .
$$

$\sigma_{\delta}$ is the relative energy spread, $\epsilon_{0}$ is the initial emittance, $\alpha$ and $\beta$ are the Twiss parameters at the end of the beam line. If the bunch length is constant, then the CSR induced $\frac{d \sigma_{\delta}}{d s}$ is constant. Hence $\frac{d \sigma_{\delta}}{d s}$ could be factored out from the integration. Therefore, if the lattice is designed such that $\int R_{16}(s) d s=0$ and $\int R_{26}(s) d s=0$, then no net emittance growth is expected, even though it may oscillate.

In a bunch compressor, either an Arc or a Chicane, the bunch length is no longer a constant, hence, $\frac{d \sigma_{\delta}}{d s}$ is no longer a constant. So, along the beam line, the CSR kicks will have different strength. The CSR kick originated at location $x$ will cause the electron to follow a betatron oscillation in the downstream beam line. Hence, the transverse emittance is modified. In periodic structure, we then could adjust the lattice design such that there will be a halfinteger phase advance ${ }^{[7]}$ between two identical cells, therefore, two CSR kicks will be $180^{\circ}$ out of phase, and this leads to the cancellation.

In Fig. 3, we demonstrate an ideal case. The particle was initially following the solid line, due to the CSR, it is kicked to the dashed line. After it traverses a cell, it meets a second kick with the same strength. If these two kicks are $(2 n+1) \pi$ in betatron phase away from each other. The second kick will bring the particle from the dashed line back to the solid line. So, the CSR induced kicks cancel with each other pair-wise. In the real case, the two kicks do not have the same strength, but as long as there is a halfinteger phase advance between them, they are $180^{\circ}$ out of phase, hence they cancel partially.

\section{PERL ARC COMPRESSOR}

In the PERL project, we use two arcs as bunch compressors ${ }^{[8]}$. In our design, the beam initial parameters are: $\sigma_{t}=3 \mathrm{ps}, \epsilon_{n}=0.5 \pi \mathrm{mm}-\mathrm{mrad}, \frac{\sigma_{\gamma}}{\gamma} \approx 9.3 \times 10^{-4}$ at $E=25 \mathrm{MeV}$, and $Q_{0}=0.15 \mathrm{nC}$. The bunch is chirped during the acceleration to $300 \mathrm{MeV}$, then it enters the small arc, where the bunch is compressed to $\sigma_{t}=0.4 \mathrm{ps}$. Since the bunch is relatively long in this compressor, the CSR effect is not significant. The transverse emittance grows $7 \%$, and $\frac{\sigma_{\gamma}}{\gamma}$ grows $3 \%$. The beam is further accelerated to $3 \mathrm{GeV}$, and then enters the big arc. For the first 90 degree, the net $R_{56}=0$, hence, there is no compression. In the remaining 90 degree, the bunch is compressed 
Two identical cells

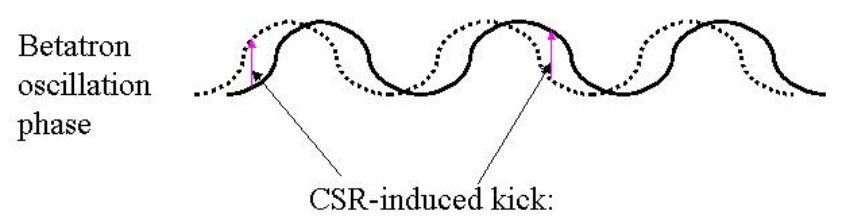

Identical, but $(2 n+1) \pi$ in betatron phase away from each other

Figure 3: Transverse emittance cancellation mechanism Half-integer phase advance.

from $0.4 \mathrm{ps}$ to $0.1 \mathrm{ps}$. In this compression, the CSR effect could be significant, easily leads to a $100 \%$ transverse emittance growth. Hence we implement the above mentioned pairwise emittance growth cancellation mechanism in the arc design, i.e., identical cells with a half-integer phase advance. Due to the small arc, the input $\epsilon_{n}$ to the big arc is about $0.533 \pi \mathrm{mm}-$ mrad. After compression, $\epsilon_{n}$ increases only to $0.543 \pi \mathrm{mm}-\mathrm{mrad}$. Such a mechanism works further in the decompression arc, where $\epsilon_{n}$ is reduced to $0.536 \pi \mathrm{mm}-\mathrm{mrad}$, almost the same as the input value. Shown in Fig. $4, \epsilon_{n}$ grows and reduces pairwisely. This ensures the energy recovery.

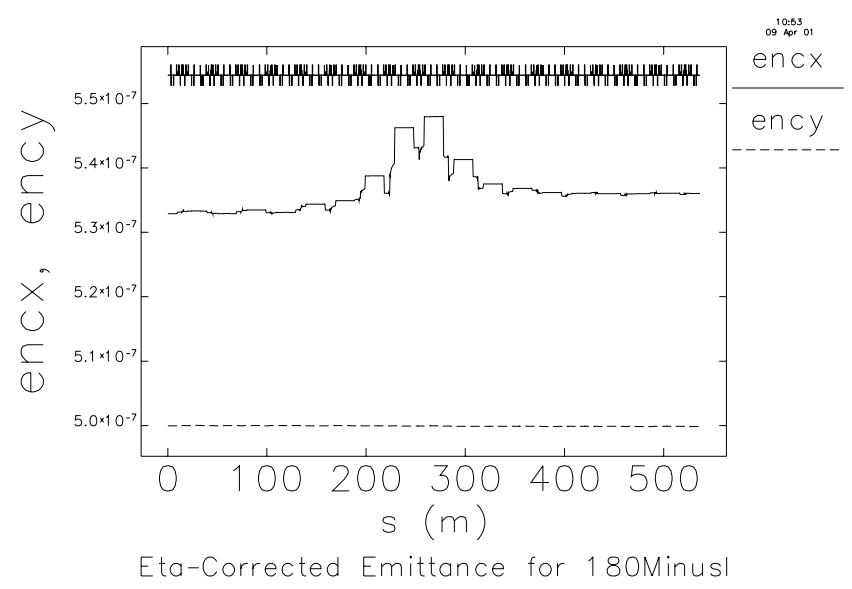

Figure 4: The variation of the transverse emittance.

\section{UVFEL CHICANE COMPRESSOR}

In the UVFEL project at the NSLS, the bunch is to be compressed from 4 ps FWHM to 1 ps FWHM at an energy of $80 \mathrm{MeV}$. In order to compress the bunch, before the chicane, we introduce a chirp in the accelerator cavity at a chirping angle $\phi$ (in our notation $\phi=0$ means on-crest). The chicane has an adjustable $R_{56}$ to match to the energy chirp. The beam has $E_{0}=44 \mathrm{MeV}$ after the first accelerator cavity, $Q_{0}=0.4 n C, \tau_{F W H M}=4 \mathrm{ps}, \frac{\sigma_{\gamma}}{\gamma}=0.1 \%$, $\epsilon_{n}=4 \pi m m-$ mrad. This beam is then accelerated to $80 \mathrm{MeV}$ in the second cavity, where a suitable amount of chirp is imposed on it. The chirped beam is then compressed in the following chicane to $\tau_{F W H M} \approx 1 \mathrm{ps}$. After that the beam is further accelerated to about $160 \mathrm{MeV}$ before entering the NISUS undulator for FEL operation, which requires $\frac{\sigma_{\gamma}}{\gamma}<\rho \sim 2.5 \times 10^{-3}$, the Pierce parameter.

A nominal set of parameters to fulfill the above requirement is the following. We chirp the bunch at about $20^{\circ}$ degree, with a corresponding bending angle of about $16^{\circ}$ degree per magnet in the chicane. In such case, the final $\frac{\sigma_{\gamma}}{\gamma}$ at $160 \mathrm{MeV}$ would be smaller than $\rho$, and $\epsilon_{n}$ grows only $15 \%$. Further consideration of adjusting the dispersive function in the chicane ${ }^{[9]}$ will be helpful.

\section{CONCLUSION}

In conclusion, the CSR is coherent, and acts like an external classical field, but not like a random quantum excitation, hence it is subject to manipulation. Possible cures are discussed in the paper. Since the CSR causes the head particles to gain energy, and the tail particles to lose energy, this is in contract to most wake fields, such as the resistive wall wake field, cancellations among the CSR effect and other wake field effects seems possible ${ }^{[10]}$. Using the above mentioned techniques, emittance growth could be made insignificant for the PERL project. In the UVFEL, careful choice of parameters will ensure the FEL operation.

\section{ACKNOWLEDGMENT}

The authors would like to thank M. Borland (ANL) for assistance with his Elegant code. The work is done under the contract $D E-A C 02-98 C H 10886$ with the US Department of Energy.

\section{REFERENCES}

[1] I. Ben-Zvi, et al., this proceedings.

[2] J.B. Murphy, S. Krinsky, and R.L. Gluckstern, PAC95, 2980 (1995); Part. Accl. 57, 9 (1997).

[3] Ya.S. Derbenev, J. Rossbach, E.L. Saldin, and V.D. Shiltsev, DESY-TESLA-FEL-95-05 (1995).

[4] Ya.S. Derbenev, and V.D. Shiltsev, SLAC-PUB-7181 (1996).

[5] M. Borland, ICAP (2000).

[6] P. Emma, and R. Brinkmann, PAC97, 1679 (1997).

[7] D. Douglas JLAB-TN-98-012 (1998).

[8] V. Yakimenko, et al., this proceedings.

[9] M. Borland (private communications).

[10] J.B. Murphy, et al., this proceedings. 